\title{
ORIENTACIÓN AL MERCADO Y EFICIENCIA EN LOS SUPERMERCADOS: EVIDENCIAS EMPÍRICAS
}

\author{
Market orientation and efficiency in supermarkets: \\ empirical evidence
}

\author{
Simone Regina Didonet ${ }^{1}$ \\ Guillermo Javier Díaz Villavicencio²
}

\begin{abstract}
Resumen
Ese trabajo analiza la relación entre orientación al mercado y eficiencia en los supermercados. El modelo de orientación al mercado fue adaptado de Kohli e Jaworski (1990). La investigación es conclusiva descriptiva y metodología incluye un cuestionario aplicado a los gerentes de supermercados y la utilización de la técnica DEA (Análisis Envolvente de Datos) y la regresión Tobit para el enálisis de los datos. Los resultados muestran que acciones específicas de generación y diseminación de inteligência y de implementación de respuesta influyen de manera positiva en los niveles de eficiencia y que la concepción de respuesta influye negativamente. Los resultados también confirman la eficiencia como una medida adecuada en la relación con la orientación al mercado y muestran que es posible trabajar con dos enfoques teóricos poco explorados en conjunto: uno originario de la teoría de marketing y otro de la teoría neoclásica de la empresa.
\end{abstract}

Palabras Clave: Orientación al Mercado; Eficiencia; Supermercados.

\begin{abstract}
This work analyzes the relationship between market orientation and efficiency in supermarkets. The model of market orientation was adapted from Kohli and Jaworski (1990). The

'Doutora em Administração (CEPEAD/UFMG), mestre em Engenharia de Produção (PPGEP/UFSM), Graduada em Administração (UNIJUI), Professora Assistente na Facultad de Economía y Administración da Universidad Católica del Norte, Chile, Av. Angamos, 0610, Antofagasta/Chile. E-mail: sdidonet@ucn.cl

${ }^{2}$ Doutorando em Estudos Empresariais, Universidad de Barcelona/Espanha, mestre em Investigação em Economia de Empresas, Universidad Autónoma de Barcelona/Espanha, Graduado em Administração de Empresas, Universidad de Antofagasta, Chile, Pasaje Las Encinas, 1630, Edificio El Sauce, 201, Parque Inglés Antofagasta, Chile. E-mail: guillermojavier.diaz@uab.es
\end{abstract}


research is conclusive and descriptive and the methodology includes a survey with managers and the use data envelopment analysis and Tobit Regression for data analysis. The results show that specific actions of generation and dissemination of intelligence and implementation of response have a positive influence on efficiency levels and that conception of response has a negative influence on it. The results confirm that efficiency is an appropriate measure in the relation with market orientation and reveals that the approach of two theoretical fields little explored in set is feasible: one from marketing theory and other from neoclassic theory of the firm.

Keywords: Market Orientation; Efficiency; Supermarkets.

\section{Introducción}

La orientación al mercado es originaria del concepto de marketing y es comprendida como la habilidad de las empresas en aprender continuamente sobre sus clientes, la competencia y el ambiente y reaccionar sobre eses eventos tanto en mercados actuales como en mercados potenciales (DAY, 1994).

La temática ha sido una discusión importante en el ámbito de la teoría de marketing desde la década de 1990 y distintas versiones surgieron a partir de los trabajos pioneros de Kohli y Jaworski (1990) y de Narver y Slater (1990). Entre las propuestas se destacan: la profundización de las cuestiones internas de orientación al mercado con enfoque en la cultura organizacional (DESPHANDÉ y WEBSTER, 1989; DESPHANDÉ, FARLEY y WEBSTER, 1993; GEBHARDT, CARPENTER y SHERRY, 2006); las capacidades internas en generar orientación al mercado (DAY y WENSLEY, 1988; DAY, 1994; DAY, 2001); el alineamiento de la orientación al mercado con la inovacción y el impacto de las dos en el desempeño (HAN, KIM Y SRIVASTAVA, 1998; LOCANDER et al., 2002; ZHOU, YIM y TSE, 2005); el alineamiento de la orientación al mercado con el aprendizaje organizacional (SLATER y NARVER, 1995; BAKER y SINKULA, 1999b; FARRELL y OCZKOWSKI, 2002); la orientación al mercado en distintos contextos y países (PITT, CARUANA y BERTHON, 1996; ROGERS, GHAURI y GEORGE, 2005).

En gran parte de esas propuestas la relación entre la orientación al mercado y el desempeño es frecuente y comprovadamente positiva, aunque excepciones deben ser consideradas. Por ejemplo las investigaciones de Baker y Sinkula (1999b), Appiah-Adu(1998) y de Antoni, Damacena y Lezana (2004), ese último realizado en Brasil, muestran que la orientación al mercado no influye en el desempeño, y Sargeant y Mohamad (1999) encontraron que la orientación al mercado influye negativamente.

Teniendo en cuenta los resultados en desacuerdo y con el intento de identificar posibles fallas en los estudios realizados hasta el momento, tres aspectos centrales pueden ser destacados: (1) las medidas de desempeño utilizadas para establecer la relación con la orientación al mercado; (2) los efectos individualizados de los factores de la orientación al mercado en el desempeño; (3) el contexto de realización de las investigaciones.

En cuanto al primer aspecto es importante considerar que las medidas de desempeño generalmente utilizadas en la relación con la orientación al mercado representan el desempeño financiero de la empresa, por ejemplo la lucratividad, el retorno sobre la inversión, el retorno sobre el activo (VENKATRAMAN y RAMANUJAM, 1986). En la clasificación de Walker y Ruekert (1987) esas serían medidas de eficiencia del negocio. Sin embargo la eficiencia puede ser por si misma una medida de desempeño (SCHENDEL y PATHON, 1978) que representa la relación 
directa entre inputs utilizados y outputs generados. Esa es la eficiencia técnica, un concepto que se origina de la función de producción en la teoría neoclásica de la firma (SALAS, 1984). Sin embargo esa medida no recibe mucha atención en los estudios sobre orientación al mercado, aunque Jaworski y Kohli (1993) hayan considerado la importancia de estudiar dimensiones alternativas de desempeño del negocio, para explorar la complejidad de la relación con la orientación al mercado. En ese sentido la eficiencia puede ser una alternativa a las medidas tradicionales de desempeño y puede revelar aspectos importantes del perfil de la empresa cuando orientada al mercado.

Con relación al segundo aspecto, las investigaciones en general buscan la relación del constructo pero no hay claridad en cuanto a cuáles factores componentes del mismo influyen más o menos en el desempeño.

En cuanto al contexto de realización de las investigaciones, gran parte de los estudios se direcciona hacia las empresas de manufactura y/o de servicios (KIRCA, JAYACHANDRAN y BEARDEN, 2005) y se pone muy poca atención en las empresas comerciales, en especial las empresas minoristas de alimentos, y entre ellas los supermercados. Las excepciones son los estudios de Rogers, Ghauri y George (2005) y Panigyrakis y Theodoridis (2007).

En investigaciones realizadas en Brasil se percibe más interés por el sector minorista y un ejemplo son los estudios de Sampaio (2000), Castilho Filho y Akel Sobrinho (2004) y Souza (2004). Sin embargo, ninguno de esos estudios centró las atenciones en el segmento de los supermercados, lo cual presenta un contexto poco explorado sobre la relación entre orientación al mercado y desempeño, enfrenta fuerte competencia, gran concentración de empresas, y busca la satisfacción de distintos grupos de clientes (PANIGYRAKIS y THEODORIDIS, 2007). Eses aspectos hacen del segmento una fuente importante para explorar el tema.

Teniendo en cuenta tales aspectos, el objetivo del presente trabajo es analizar la relación entre los componentes de la orientación al mercado y la eficiencia en el segmento de supermercados. La investigación fue realizada en una muestra de supermercados que actúan en la ciudad de Belo Horizonte, Minas Gerais, Brasil.

La estructura del trabajo es la siguiente: el apartado 2 presenta la teoría sobre orientación al mercado y eficiencia y las hipótesis de investigación; el apartado 3 presenta la metodología utilizada; en el apartado 4 están los resultados; en el apartado 5 están las conclusiones de estudio; el trabajo termina con las referencias utilizadas.

\section{Marco teórico e hipótesis de investigación orientación al mercado}

La orientación al mercado se originó filosóficamente del concepto de marketing (JAWORSKI y KOHLI, 1993) y creció como teoría en la década de 1990. Sin embargo, ya en la década de 1980 hubo una renovación del interés por el concepto y por la filosofía de marketing. Webster (1988) habla del re descubrimiento del concepto en un periodo donde la competencia y la complejidad de los mercados aumentaron considerablemente. En esa época el término orientación al mercado empezó a ser utilizado como sinónimo del concepto de marketing (SHAPIRO, 1988). Soportes conceptuales integradores se destacaron en esa época y se mantuvieron hasta la primera mitad de los años 1990, cuando eran frecuentes las discusiones sobre fuentes de ventaja competitiva sostenible, implementación de estrategias de negocios, marketing relacional, control de marketing, procesos de innovación y diseño de productos (KERIN, 1996).

La propuesta de destacar el concepto de orientación al mercado marcó definitivamente la década de 1990, cuando teorías comprensivas sobre el constructo, su naturaleza y sus

Rev. Adm. UFSM, Santa Maria, v. I, N. 1, P. 134-152, Jan./ABR. 2008 
consecuencias fueron desarrolladas y surgió un grupo de investigaciones sobre la relación entre orientación al mercado y desempeño (SLATER y NARVER, 1995).

Argumentando que la literatura necesitaba una definición clara de orientación al mercado y que los practicantes necesitaban de descripciones específicas sobre el tema y de su actual efecto sobre el desempeño, Kohli y Jaworski (1990) y Narver y Slater (1990), propusieron una agenda de investigación y surgieron como los primeros autores a discutir empíricamente el asunto. Desde entonces, las investigaciones se ampliaron para distintos contextos y países. Con una propuesta de comprender la cultura orientada hacia el mercado, Desphandé, Farley y Webster (1993) y Day (1994) también son considerados pioneros de estudios de esa naturaleza.

En la concepción y definición del constructo, se evidencian distintas percepciones sobre los elementos de dominio de la orientación al mercado. Para Kohli y Jaworski (1990:6) "orientación al mercado es la amplia generación de inteligencia de mercado referente a las necesidades de los actuales y futuros clientes, la diseminación de la inteligencia a través de los sectores de la empresa y la amplia respuesta a ellas." Los autores también consideran que el constructo es influenciado por aspectos como la participación de los directores/gerentes y por los conflictos departamentales, es moderado por variables ambientales (turbulencia tecnológica, turbulencia de mercado e intensidad competitiva) y se manifiesta en el espíritu del equipo de la empresa y en sus resultados.

Por otro lado, Narver y Slater (1990) consideran que la orientación al mercado se compone de tres aspectos: (1) orientación al cliente, caracterizada por el entendimiento de los clientes y por la habilidad en crear valor superior a ellos; (2) la orientación a la competencia, que consiste en el entendimiento de las fuerzas, debilidades y capacidades de la competencia actual y potencial; (3) coordinación entre funciones, representada por la utilización coordinada de los recursos de la empresa para generar valor superior a los clientes.

Discutiendo los temas relacionados a la cultura de la empresa, Day (2001:19) percibe la orientación al mercado como "una capacidad superior para comprender, atraer y mantener clientes importantes". De acuerdo con el autor, se alcanza esa capacidad por medio de la combinación de tres elementos fundamentales: (1) una cultura orientada hacia afuera; (2) aptitudes específicas; (3) tener una estructura que permita generar valor superior al cliente, adelantarse a las condiciones del mercado y responder a ellas.

En la misma línea de Day (2001), los autores Desphandé, Farley y Webster (1993) revelaron la importancia de considerar las cuestiones culturales y de la innovación en la orientación al mercado, hecho explorado por otros autores, como Gebhardt, Carpenter y Sherry (2006), Han, Kim y Srivastava (1998), Hooley, Lynch y shepherd (1990).

En común, gran parte de las propuestas indican la relación positiva entre la orientación al mercado y el desempeño, comprobada por investigaciones empíricas realizadas en distintos sectores industriales. También otros estudios mostraron que la orientación al mercado no influye en el desempeño, como por ejemplo las investigaciones de Baker y Sinkula (1999b), Appiah-Adu (1998) y de Antoni, DamacenA y Lezana (2004), ese último realizado en Brasil, y la investigación de Sargeant y Mohamad (1999) que mostró una influencia negativa. Tal vez las diferencias en los resultados estén relacionadas al contexto y al perfil de las empresas estudiadas y a las medidas de desempeño utilizadas. Con relación a dichas medidas, Jaworski y Kohli (1993) destacan la importancia de considerar dimensiones alternativas de desempeño del negocio, para explorar la complejidad de la relación con la orientación al mercado.

Siguiendo esa lógica se propone utilizar la eficiencia técnica como la medida de desempeño en el presente trabajo y explorar la relación con la orientación al mercado en el sector minorista de alimentos, donde las medidas ampliamente utilizadas en marketing son 
la participación del mercado (DENG y DART, 1994; LIU y DAVIES, 1997; PANIGYRAKIS y THEODORIDIS, 2007), la productividad del trabajo (INGENE, 1982), el volumen y el crecimiento de las ventas (PANIGYRAKIS y THEODORIDIS, 2007; SAMPAIO, 2000).

\section{Eficiencia técnica}

Las cuestiones sobre eficiencia se originaron de la teoría neoclásica de la firma, donde la empresa era considerada una unidad elementar de la producción, ejerciendo una función de demandante de factores productivos y creadora de bienes y servicios (SALAS, 1984).

Los primeros trabajos que trataron de la eficiencia y de sus medidas son atribuidos a Koopmans (1951) y a Debreu (1951), pero fue Farrell (1957) que definió la frontera eficiente y propuso la descomposición de la eficiencia en dos partes: eficiencia técnica y eficiencia de precio. Fare, Grosskopf y Lovell (1983) siguieron la orientación de Farrell (1957) y propusieron la descomposición en tres componentes: eficiencia técnica global; eficiencia técnica pura y eficiencia de escala. En el apartado sobre la metodología se presentan las formulaciones específicas de la eficiencia que fueron utilizadas en el presente trabajo.

Partiendo de las consideraciones sobre los constructos que están siendo analizados y teniendo en cuenta el objetivo de analizar la relación entre orientación al mercado y eficiencia, se proponen las hipótesis de investigación en el apartado siguiente.

\section{Hipótesis de investigación}

En términos del constructo orientación al mercado y de su efecto sobre el desempeño, Narver y Slater (1990), Jaworski y Kohli (1993), Slater y Narver (1994), Day (2001) y Han, Kim y Srivastava (1998), entre otros, comprueban la relación positiva entre ambos. Panigyrakis y Theodoridis (2007) lo comprueban también en un estudio realizado en los supermercados. Teniendo en cuenta eses hallazgos y considerando la eficiencia como medida de desempeño (SCHENDEL y PATHON, 1978) y la descomposición de la orientación al mercado en generación de inteligencia, diseminación de inteligencia, concepción de respuesta e implementación de respuesta (KOHLI y JAWORSKI, 1990), las hipótesis que serán probadas son las siguientes:

\subsection{Ceneración de inteligencia}

$\mathrm{H}_{1}$ : A mayor obtención de informaciones de los clientes, mayor será el nivel de eficiencia de los supermercados.

$\mathrm{H}_{2}$ : A mayor obtención de sugerencias del mercado, mayor será el nivel de eficiencia de los supermercados.

$\mathrm{H}_{3}$ : A mayor obtención de informaciones del segmento de actuación, mayor será el nivel de eficiencia de los supermercados.

$\mathrm{H}_{4}$ : A mayor percepción de los cambios en el segmento, mayor será el nivel de eficiencia de los supermercados.

La proposición de esas hipótesis fue basada en el hecho de que la generación de la inteligencia involucra el conocimiento de las necesidades y preferencias de los clientes e incluye aspectos como encuestas con clientes, discusiones con clientes y socios, y el análisis de factores relacionados a la competencia y al ambiente de negocios de la empresa (KOHLI y JAWORSKI, 1990). 


\subsection{Diseminación de inteligencia}

La diseminación de inteligencia se viabiliza por medio de la comunicación horizontal que debe ocurrir entre los departamentos de la empresa (KOHLI y JAWORSKI, 1990), cuando las necesidades del mercado deben ser comunicadas (McNAMARA, 1972). Todos los miembros de la empresa deben estar comprometidos en crear valor superior a los clientes (NARVER, SLATER y TIETJE, 1998). En tal sentido, las hipótesis que serán probadas son las siguientes:

$\mathrm{H}_{5}$ : A mayor intercambio de informaciones entre los departamentos sobre el mercado, mayor será el nivel de eficiencia de los supermercados.

$\mathrm{H}_{6}$ : A mayor discusión de las necesidades futuras de los clientes entre los departamentos, mayor será el nivel de eficiencia de los supermercados.

$\mathrm{H}_{7}$ : A mayor cambio de informaciones de los clientes, mayor será el nivel de eficiencia de los supermercados.

\subsection{Concepción de respuesta}

De acuerdo con Kohli y Jaworski (1990), la concepción de respuesta consiste en la utilización de la inteligencia de mercado para desarrollar las acciones en respuesta al mercado, que están relacionadas a: selección de mercados, diseño y oferta de productos en respuesta a las necesidades actuales y futuras de los clientes. En tal sentido, las hipótesis que serán probadas son las siguientes:

$\mathrm{H}_{8}$ : A mayor aceptación de los cambios en las necesidades de los clientes, mayor será el nivel de eficiencia de los supermercados.

$\mathrm{H}_{9}$ : A mayor relación de los planes con los cambios de mercado, mayor será el nivel de eficiencia de los supermercados.

$\mathrm{H}_{10}$ : A mayor definición de los planes de acuerdo con la realidad del segmento, mayor será el nivel de eficiencia de los supermercados.

$\mathrm{H}_{11}$ : A mayor reacción a los cambios de precios de la competencia, mayor será el nivel de eficiencia de los supermercados.

$\mathrm{H}_{12}$ : A mayor oferta de productos y de servicios que sigue la segmentación de mercado, mayor será el nivel de eficiencia de los supermercados.

\subsection{Implementación de respuesta}

La implementación de respuesta se relaciona a la consecución de los planes definidos en la concepción de respuesta y junto a esa, define la respuesta de la empresa al mercado. En la implementación de respuesta son ejecutadas las acciones referentes a la selección del mercado y a la oferta de productos y servicios para responder a las necesidades de los clientes (JAWORSKI y KOHLI, 1993). En tal sentido se propone las siguientes hipótesis: eficiencia.

$\mathrm{H}_{13}$ : A mayor reacción de los supermercados a la competencia, mayor será el nivel de

$\mathrm{H}_{14}$ : A mayores acciones en contra a la insatisfacción de los clientes, mayor será el nivel de eficiencia de los supermercados.

$\mathrm{H}_{15}$ : A mayor oferta de nuevos servicios, mayor será el nivel de eficiencia de los supermercados.

$\mathrm{H}_{16}$ : A mayores acciones para establecer relaciones con los clientes, mayor será el nivel de eficiencia de los supermercados. 
En la secuencia se presenta la metodología que fue utilizada para el desarrollo de la investigación.

\section{Metodología}

La investigación es conclusiva descriptiva (MATTAR, 1999) y el método utilizado fue el survey (MALHOTRA, 2001 y BABBIE, 1999). La recogida de datos se hizo por medio de cuestionarios respondidos por los gerentes de supermercados de Belo Horizonte, Minas Gerais, y que son afiliados a la Cámara de Comercio de la ciudad.

La muestra fue por conveniencia (MALHOTRA, 2001) y fueron consultados 418 supermercados de los cuales 119 se propusieron a responder el cuestionario. De eses, 15 supermercados fueron identificados al azar para el estudio piloto. La estrategia utilizada para aplicar el cuestionario fue la encuesta personal (MALHOTRA, 2001) y el cuestionario fue dividido en dos partes: la primera referente a la orientación al mercado y elaborada con escala Likert de 1 a 5, adaptada de Kohli y Jaworski (1993); la segunda con cuestiones referentes a los datos necesarios para el cálculo de la eficiencia de las empresas. La recogida de datos ocurrió entre los meses de septiembre y noviembre de 2006. Para la validación de contenido se hizo paneles de especialistas, dos pre pruebas y un estudio piloto (BABBIE, 1999). Para la fiabilidad del cuestionario se hizo la prueba del alfa de Cronbach (MALHOTRA, 2001). La muestra final, eliminados los cuestionarios con datos omitidos y los casos que hicieron parte del estudio piloto, quedó en 67 casos. Luego, la tasa de respuesta fue de 64,4\% (considerando los casos investigados), y está de acuerdo con investigaciones previas en el área, realizadas con muestras semejantes a la muestra de la presente investigación: 77\% de respuesta para Phillips, Davies y Moutinho (2002), 58\% para Appiah-Adu y Ranchhod (1998) y 43\% para Sargeant y Mohamad (1999). Es importante destacar que el mayor índice de no respuesta ocurrió en las cuestiones necesarias al cálculo de la eficiencia, que es la variable dependiente de la regresión, y por lo tanto se hizo la opción por la eliminación de los casos siguiendo la orientación de Hair, Anderson y Tatham (1998).

El análisis de los datos se hizo en dos etapas. Para la primera etapa fue aplicada la técnica DEA (Data Envelopment Analysis) para la definición de los coeficientes de eficiencia de los supermercados y se hizo el análisis factorial para la definición de los componentes de la orientación al mercado. Para la segunda etapa se hizo la regresión de Tobit para verificar la relación entre las variables de orientación al mercado y la eficiencia. También se hizo la prueba de correlación de Pearson para las variables independientes.

En cuanto al DEA, es una técnica de análisis no paramétrica que permite evaluar la eficiencia relativa de unidades individuales (DMUs - Decision Making Units) dentro de una dicha población, por medio de la definición de una frontera eficiente (CHARNES, COOPER y RHODES, 1978). Las DMUs son consideradas eficientes cuando obtienen el resultado óptimo de la relación outputs/inputs. Para tanto se consideran dos situaciones: (1) cuando ninguna otra DMU puede producir un nivel superior de outputs con la misma cantidad de input; (2) cuando ninguna otra $D M U$ puede emplear un menor nivel de inputs para producir la misma cantidad de outputs. En el primer caso, la orientación es para los outputs y en el según caso es para los inputs. La opción por la orientación se hace teniendo en cuenta las condiciones operacionales del sector o del segmento evaluado. En ese sentido se resume en la secuencia las ventajas y desventajas de cada caso.

La orientación a los inputs implica la máxima reducción posible de los inputs para producir la misma cantidad de outputs. Teniendo en cuenta el tema bajo investigación, disminuir las cantidades de inputs podría significar la reducción de los esfuerzos de los supermercados

Rev. Adm. UFSM, Santa Maria, v. I, N. 1, P. 134-152, Jan./ABr. 2008 
hacia la comprensión de las necesidades de los clientes. Aunque el contexto del segmento sea definido por bajos márgenes de ganancias y por la necesidad de disminuir costes (KEH y PARK, 1997), disminuir la cantidad de inputs significaría la indisponibilidad de recursos necesarios a las acciones de orientación al mercado y significaría no explorar el potencial de crecimiento de las empresas. Es así por que los inputs considerados en la investigación fueron el número de funcionarios, el número de cajeros y el área de ventas, conforme indicaciones de Dubelaar, Bhargava y Ferrarin (2002), Ingene (1982), Donthu y Yoo (1998).

Por otro lado, la orientación a los outputs asume una situación de aumento de las cantidades de los resultados evaluados a partir de los recursos empleados. En el caso de los supermercados y de la presente investigación, los outputs utilizados para el análisis de eficiencia fueron el número medio de clientes y el número medio de productos en stock, de acuerdo con indicación de Dubelaar, Bhargava y Ferrarin (2002), Donthu y Yoo (1998), Keh y Chu (2003). Explorar dichos outputs al máximo significa admitir que hay potencial de la empresa para garantizar su espacio en el ambiente competitivo del segmento. Esa situación es adecuada cuando son considerados los aspectos de orientación al mercado, cuya teoría destaca su potencial para generar ventaja competitiva sustentable (DAY, 2001; HUNT y MORGAN, 1995). A partir de los inputs, el aumento del número de clientes atendidos y de la variedad de productos ofrecidos por el supermercado puede reflejar acciones de la orientación al mercado. Teniendo en cuenta la gran competencia del segmento, el aumento de los referidos outputs puede ser un factor crítico de suceso para el negocio.

En cuanto a la literatura sobre eficiencia en los supermercados, no hay una tendencia por una u otra orientación lo que refuerza el argumento de que la opción refleja la realidad en estudio. Por ese motivo se hizo la opción por la orientación hacia los outputs en la presente investigación. En cuanto a la tecnología se hizo la opción por los retornos variables a la escala (VRS - Variable Return on Scale - VRS) debido a la característica poco homogénea de la muestra, principalmente debido al área de ventas que es uno de los factores representativos del tamaño de la empresa (DUBELAAR, BHARGAVA y FERRARIN, 2002).

Teniendo en cuenta esas definiciones, se obtiene la eficiencia de los supermercados por medio de la resolución del siguiente problema de programación lineal, adaptado de Fare, Grosskopf y Lovell (1994):

$\operatorname{Max} \beta$

S.t.

$$
\begin{aligned}
& \sum_{j=1}^{N} \lambda_{j} y_{s j} \geq \beta y_{s i} \quad s=1, \ldots, S \\
& \sum_{j=1}^{N} I_{j} X_{m j} \leq X_{m i} \quad m=1, \ldots M \\
& \sum^{M} \lambda_{j}=1 \\
& \lambda_{j} \geq 0 \quad j=1, \ldots N
\end{aligned}
$$

Donde:

$\beta=$ variable escalar que representa el coeficiente de eficiencia que es padrón para comparación;

$\lambda_{j}=$ vector de constantes $\mathrm{Jx} 1$;

xmj $=$ matriz de inputs Mxj; $y$

$y_{s j}=$ matriz de outputs SxJ. 
También en la primera etapa se hizo el análisis factorial por medio del método de componentes principales con rotación varimax (MALHOTRA, 2001). Avlonitis y Gounaris (1999) utilizaron el mismo criterio en la primera etapa de su estudio cuyo objetivo fue identificar los determinantes de la orientación al mercado.

Para la segunda etapa se hizo la regresión Tobit que fue desarrollada para investigaciones y estudios económicos por James Tobin, en el año de 1958 y es considerada un modelo híbrido entre el análisis Probit y el análisis de regresión múltiple (TOBIN, 1958).

El modelo de regresión Tobit utilizado para la presente investigación fue adaptado de CHOU (1999) y se presenta en la secuencia:

$$
Y^{*}=\beta_{0}+\beta_{i} x_{i}+\varepsilon \quad i=1, \ldots, n
$$

Donde:

$\mathrm{Y}^{*}=$ coeficientes de eficiencia de los supermercados (variable dependiente);

$\beta_{0}$ e $\beta_{i}=$ constantes;

$x_{i}=$ variables de orientación al mercado (variables independientes);

$\varepsilon=$ error aleatorio.

La regresión Tobit es la técnica más frecuentemente utilizada en análisis de segunda etapa de $D E A$, cuando el objetivo es analisar la relación entre los coeficientes de eficiencia y las variables explicativas (HOFF, 2006) y es una alternativa a la regresión del tipo OLS (Ordinary Least Squares). Hoff (2006) compara los dos abordajes de segunda etapa y concluye que, aunque las dos tengan restricciones en términos de los resultados, el modelo OLS parece apresentar errores de especificación más facilmente que el Tobit.

\section{Presentación y análisis de los datos eficiencia y factores de orientación al mercado de los supermercados investigados}

La eficiencia fue calculada por medio de la técnica DEA utilizándose el software Efficiency Measurement System (EMS) y siguiendo la opción por los rendimientos variables a la escala $(V R S)$ y orientación a los outputs.

Teniendo en cuenta los 67 casos válidos para el análisis, se presentan los resultados obtenidos aplicándose la fórmula [3.1] en la TAB.1 (página siguiente). Para efectos de comparación, se hizo la opción por mostrar los tamaños de las tiendas (superficie de ventas en $\mathrm{m}^{2}$ ) juntamente con los niveles de eficiencia.

Los resultados de la TAB.1 muestran 17 DMUs eficientes, lo que representa 27,4\% del total de empresas analizadas. Tomando el ejemplo de la DMU 1 es posible concluir que esa empresa es ineficiente en uno 58\%, o sea, considerando su cantidad de inputs, necesita aumentar la cantidad de outputs en uno 58\% para ser eficiente, cuando comparada con las unidades de referencia que están en la frontera eficiente.

Se percibe que la eficiencia de los casos analizados se concentra en los supermercados de pequeño tamaño (hasta $250 \mathrm{~m}^{2}$ ) y tamaño medio (de $251 \mathrm{~m}^{2}$ a $1000 \mathrm{~m}^{2}$ ), lo que suma 15 DMUs del total de 17 eficientes, hecho que puede ser considerado normal cuando se considera que gran parte de los supermercados evaluados presenta eses tamaños. Sin embargo, aunque 
la concentración de casos eficientes esté en los pequeños y medianos, no es posible afirmar categóricamente que el tamaño sea un hecho determinante de los resultados encontrados pues no fue hecha ninguna prueba para esa finalidad.

Tabla 1 - Resultados de eficiencia de los supermercados analizados

\begin{tabular}{|c|c|c|c|c|c|c|c|c|}
\hline Caso/DMU & Sup.Ventas $\left(\mathbf{m}^{2}\right)$ & Ef_VRS & Caso/DMU & Sup.Ventas $\left(\mathbf{m}^{2}\right)$ & Ef_ VRS & Caso/DMU & Sup.Ventas $\left(\mathbf{m}^{2}\right)$ & Ef_ VRS \\
\hline 1 & 400 & 1,58 & 24 & 160 & 1,81 & 47 & 620 & 1,46 \\
2 & 600 & 1,31 & 25 & 280 & 1,19 & 48 & 100 & 1,00 \\
3 & 200 & 1,35 & 26 & 150 & 1,00 & 49 & 860 & 1,40 \\
4 & 360 & 1,00 & 27 & 180 & 1,97 & 50 & 100 & 1,00 \\
5 & 400 & 1,77 & 28 & 370 & 1,00 & 51 & 700 & 1,75 \\
6 & 272 & 1,00 & 29 & 430 & 1,00 & 52 & 400 & 1,69 \\
7 & 150 & 1,00 & 30 & 1100 & 1,67 & 53 & 1800 & 1,62 \\
8 & 600 & 1,79 & 31 & 600 & 1,00 & 54 & 700 & 1,05 \\
9 & 680 & 1,13 & 32 & 110 & 1,05 & 55 & 550 & 1,60 \\
10 & 1880 & 1,35 & 33 & 1310 & 1,00 & 56 & 472 & 1,41 \\
11 & 1400 & 1,05 & 34 & 400 & 1,32 & 57 & 1800 & 1,09 \\
12 & 560 & 1,60 & 35 & 685 & 1,00 & 58 & 1700 & 1,12 \\
13 & 400 & 1,34 & 36 & 8000 & 1,00 & 59 & 200 & 1,32 \\
14 & 400 & 1,79 & 37 & 360 & 1,23 & 60 & 300 & 1,00 \\
15 & 480 & 1,24 & 38 & 400 & 1,11 & 61 & 180 & 1,58 \\
16 & 200 & 1,39 & 39 & 130 & 1,00 & 62 & 1000 & 1,00 \\
17 & 110 & 1,00 & 40 & 700 & 1,52 & 63 & 2200 & 1,10 \\
18 & 180 & 1,52 & 41 & 600 & 1,81 & 64 & 2500 & 1,15 \\
19 & 260 & 1,49 & 42 & 420 & 1,16 & 65 & 400 & 1,60 \\
20 & 230 & 1,49 & 43 & 850 & 1,14 & 66 & 300 & 1,12 \\
21 & 100 & 1,10 & 44 & 1175 & 1,10 & 67 & 1427 & 1,26 \\
22 & 250 & 1,20 & 45 & 196 & 1,02 & ------- & ------- & ------- \\
23 & 270 & 1,38 & 46 & 200 & 1,00 & ------ & ---- & ------ \\
\hline
\end{tabular}

Fuente: datos de la investigación

En los estudios sobre orientación al mercado se considera que el tamaño influye en el desempeño y es un moderador de la relación entre orientación al mercado y el desempeño. Narver y Slater (1990) y Jaworski y Kohli (1993) consideraron ese aspecto en sus investigaciones, aunque esa discusión no fue profundizada en otros estudios (GREENLEY, 1995).

En cuanto al análisis factorial, se hizo cuatro análisis, una para cada elemento de la orientación al mercado: generación de inteligencia, diseminación de inteligencia, concepción de respuesta e implementación de respuesta, eses dos últimos representando la llamada responsividad (KOHLI y JAWORSKI, 1990). Se hizo la opción por realizar análisis individuales (MALHOTRA, 2001) pues el número de factores que deberían ser extraídos era conocido.

Antes del análisis factorial fue estimado el alfa de Cronbach para cada uno de los respectivos grupos. Los valores encontrados variaron de 0,57 a 0,77 y son considerados adecuados para la investigación, según Nunnally (1970). Otros estudios de marketing presentan valores semejantes como es el caso de las investigaciones de Best, Hawkins y Albaum (1976) y de Darden y Perreault Jr. (1976). En la temática de orientación al mercado, Slater y Narver (1994) también encontraron confiabilidades entre 0,54 y 0,90. Es importante destacar que en la presente investigación esos valores fueron obtenidos por medio de la purificación de la escala (HAIR, ANDERSON y TATHAM (1998), cuando fueron eliminados algunos indicadores (PÉREZ, 2001). Otros autores que investigaron esa temática también utilizaron ese procedimiento, como es el caso de Kumar, Subramanian y Yauger (1998) y Mello y Souza (2003). El Cuadro 1 muestra la composición de los factores extraídos, juntamente con los valores de la prueba de Kaiser-Meyer-Olkin (KMO) para cada grupo. 


\begin{tabular}{|l|l|}
\hline \multicolumn{1}{|c|}{ Factores/KMO } & \multicolumn{1}{c|}{ Indicadores } \\
\hline $\begin{array}{l}\text { Generación de inteligencia } \\
(0,729)\end{array}$ & $\begin{array}{l}\text { Información de clientes } \\
\text { Sugerencias del mercado } \\
\text { Información del sector } \\
\text { Percepción de los cambios en el segmento }\end{array}$ \\
\hline $\begin{array}{l}\text { Diseminación de inteligencia } \\
(0,669)\end{array}$ & $\begin{array}{l}\text { Intercambio de info entre los deptos sobre la competencia/segmento } \\
\text { Discusiones sobre las neces futuras de los clientes entre los deptos } \\
\text { Intercambio de info sobre los clientes }\end{array}$ \\
\hline $\begin{array}{l}\text { Concepción de respuesta } \\
(0,534)\end{array}$ & $\begin{array}{l}\text { Aceptación de los cambios en las necesidades de los clientes } \\
\text { Acciones adaptadas a los cambios } \\
\text { Planes de acuerdo con la realidad del segmento } \\
\text { Reacción a las acciones de la competencia } \\
\text { Oferta sigue la segmentación del mercado }\end{array}$ \\
\hline $\begin{array}{l}\text { Implementación de respuesta } \\
(0,548)\end{array}$ & $\begin{array}{l}\text { Reacción a las acciones de la competencia } \\
\text { Acciones en contra la insatisfación de los clientes } \\
\text { Oferta de nuevos servicios } \\
\text { Programas de relación con los clientes }\end{array}$ \\
\hline
\end{tabular}

Fuente: datos de la investigación

Cuadro 1 - Elementos de la orientación al mercado en los supermercados analizados

Como referido en el Cuadro 1, los valores referentes a la adecuación de la muestra quedan entre 0,534 y 0,729. Tanto Hair, Anderson y Tatham (1998) como Malhotra (2001) consideran que valores entre 0,5 e 1,0 indican que el análisis factorial es adecuado. Llaman la atención los resultados del Cuadro 1 en cuanto a la presencia del aspecto competencia, especialmente en los factores de concepción de respuesta e implementación de respuesta. Como destacan Lynch, Keller y Ozment (2000), la competitividad es intensa en el segmento minorista de alimentos, lo que se puede percibir en los resultados. También surgen en los grupos varios aspectos relativos a los clientes, lo que permite suponer que los supermercados presentan características de orientación al mercado. De hecho, tanto en la generación y en la diseminación de la inteligencia como en la concepción y en la implementación de respuesta se evidencia la preocupación en comprender los clientes.

De acuerdo con Shapiro (1988), una empresa solamente puede ser orientada hacia el mercado si ella lo comprende. En la muestra estudiada es posible percibir esa tendencia pues más allá de la preocupación con los clientes también están presentes los aspectos relacionados al segmento de actuación. En cuanto a ese aspecto se evidencia muy intensamente el perfil competitivo del segmento que está presente en las acciones internas desarrolladas por los supermercados para responder al mercado.

\section{La influencia de la orientación al mercado en los niveles de eficiencia de los supermercados}

A partir del análisis factorial se hizo el análisis de regresión teniendo las variables de orientación al mercado como variables independientes. Antes de la regresión se hizo en análisis de correlación con el objetivo de verificar la existencia de multicolinealidad. Los resultados del coeficiente de correlación de Pearson mostraron que ninguna de las variables presenta correlación superior a 0,6, lo que "no presenta un problema para los análisis de regresión siguientes" (LIN y CHEN, 2005:163).

Los resultados de la regresión de Tobit, realizada con el auxilio del software Stata 9.1 y aplicándose la ecuación [3.2], son presentados en la TAB. 2 (página siguiente). 
Es importante destacar que los valores fueron censurados a la izquierda y a la derecha dentro del límite de los resultados de los coeficientes resultantes del DEA. También se hizo la opción por la regresión de cada uno de los indicadores resultantes del análisis factorial de manera independiente, al contrario de lo que normalmente se hace en los estudios sobre orientación al mercado.

De acuerdo con los resultados de la regresión, el modelo es significativo (Prob>chi2 = 0,0239), a un nivel de confianza superior a 95\%. Ese resultado permite inferir que la orientación al mercado y la eficiencia se ajustan como modelo. Luego, la eficiencia puede ser considerada una medida alternativa de desempeño en la relación y eso va al encuentro de la sugerencia de Jaworski y Kohli (1993:65) de que "sería útil explorar las complejidades de la relación entre orientación al mercado y dimensiones alternativas de desempeño del negocio en futuros estudios".

Los resultados también muestran que cuatro variables son significativas en la relación con la eficiencia: una referente a la generación de inteligencia (gi_2); una relativa a la implementación de respuesta (ir_4); una relativa a la diseminación de inteligencia (di_2); y una relativa a la concepción de respuesta (cr_2). Los coeficientes negativos de las variables gi_2, ir_4 y di_2 indican una relación positiva con la eficiencia, una vez que la ineficiencia es eliminada a cada unidad que es aumentada de las referidas variables; o sea, la eficiencia es mejorada. Se hace la interpretación al contrario por que en el caso de la orientación al ouput, las DMUs ineficientes presentan valores superiores a 1 y necesitan disminuir la ineficiencia para llegar a la frontera. En el caso de la $D M U_{1}$, referida en la TAB. 1, significa que es necesario disminuir su ineficiencia en 0,58 llegar a la frontera. Luego, la interpretación al inverso es necesaria para los resultados. En tal sentido el coeficiente positivo de la variable cr_2 muestra una relación negativa de esa con la eficiencia, lo que sugiere que la ineficiencia aumenta a cada unidad aumentada de dicha variable.

Tabla 2 - Relación de la orientación al mercado con la eficiencia de los supermercados

\begin{tabular}{|c|c|c|}
\hline \multicolumn{2}{|c|}{ Regresión Tobit } & $\begin{array}{l}\text { Número de Obs. }=67 \\
\text { Prob }>\text { chi2 }=0.0239\end{array}$ \\
\hline eff & Coef. & $P>t$ \\
\hline gi_1 & -.0565016 & 0.217 \\
\hline gi_2 & -.0916281 & $0.092^{*}$ \\
\hline gi_3 & .0132586 & 0.797 \\
\hline gi_4 & -.0134341 & 0.763 \\
\hline ir_1 & .0073918 & 0.907 \\
\hline ir_2 & -.0409553 & 0.287 \\
\hline ir_3 & .0561778 & 0.226 \\
\hline ir_4 & -.1227773 & $0.006 * * *$ \\
\hline di_1 & -.0067305 & 0.887 \\
\hline di_2 & -.0913411 & $0.074 *$ \\
\hline di_3 & -.0216195 & 0.645 \\
\hline Cr_1 & -.0658609 & 0.146 \\
\hline cr_2 & .1373336 & $0.024 * *$ \\
\hline cr_3 & .0419464 & 0.378 \\
\hline Cr_4 & .0262958 & 0.531 \\
\hline cr_5 & -.0386071 & 0.341 \\
\hline
\end{tabular}

Fuente: Datos de la investigación. 
En cuanto a la prueba de hipótesis, los resultados son mostrados en el Cuadro 2 (página siguiente).

Por los resultados mostrados en el Cuadro 2 es posible inferir que las acciones de obtención de informaciones del mercado $\left(\mathrm{H}_{2}\right)$, que la discusión de las necesidades futuras de los clientes entre los departamentos $\left(\mathrm{H}_{6}\right)$ y que las acciones que tratan de establecer programas de relación con los clientes $\left(\mathrm{H}_{16}\right)$ interfieren positivamente en la eficiencia de los supermercados. En tal sentido, a la medida que esas acciones son intensificadas, mejores serán los niveles de eficiencia de las empresas. La variable de la hipótesis $\mathrm{H}_{9}$ también mostró influencia en la eficiencia pero con impacto negativo, indicando que cuanto más los planes estuvieran relacionados con los cambios del mercado, mayor será la ineficiencia de los supermercados. Las otras hipótesis no mostraron significancia en la relación, lo que permite inferir que la relación entre la orientación al mercado y la eficiencia en los supermercados es de intensidad relativamente baja.

Cuadro 2 - Resultado de la prueba de hipótesis en la relación entre orientación al

\begin{tabular}{|c|c|c|}
\hline Grupo & Descripción de las hipótesis & Resultado \\
\hline $\begin{array}{l}\text { Generación de } \\
\text { inteligencia }\end{array}$ & $\begin{array}{l}\text { H1- A mayor obtención de informaciones de los clientes, mayor será el nivel } \\
\text { de eficiencia de los supermercados } \\
H_{2}-\text { A mayor obtención de sugerencias del mercado, mayor será el nivel de } \\
\text { eficiencia de los supermercados } \\
\mathrm{H}_{3} \text { - A mayor obtención de informaciones del segmento de actuación, mayor } \\
\text { será el nivel de eficiencia de los supermercados } \\
\mathrm{H}_{4} \text { - A mayor percepción de los cambios en el segmento, mayor será el nivel } \\
\text { de eficiencia de los supermercados }\end{array}$ & $\begin{array}{l}\text { Rechazada } \\
\text { Confirmada } \\
\text { Rechazada } \\
\text { Rechazada }\end{array}$ \\
\hline $\begin{array}{l}\text { Diseminación de } \\
\text { inteligencia }\end{array}$ & $\begin{array}{l}\mathrm{H}_{5} \text { - A mayor intercambio de informaciones entre los departamentos sobre el } \\
\text { mercado, mayor será el nivel de eficiencia de los supermercados } \\
\mathrm{H}_{6}-\text { A mayor discusión de las necesidades futuras de los clientes entre los } \\
\text { departamentos, mayor será el nivel de eficiencia de los supermercados } \\
\mathrm{H}_{7}-\text { A mayor cambio de informaciones de los clientes, mayor será el nivel de } \\
\text { eficiencia de los supermercados }\end{array}$ & $\begin{array}{l}\text { Rechazada } \\
\text { Confirmada } \\
\text { Rechazada }\end{array}$ \\
\hline $\begin{array}{l}\text { Concepción de } \\
\text { respuesta }\end{array}$ & 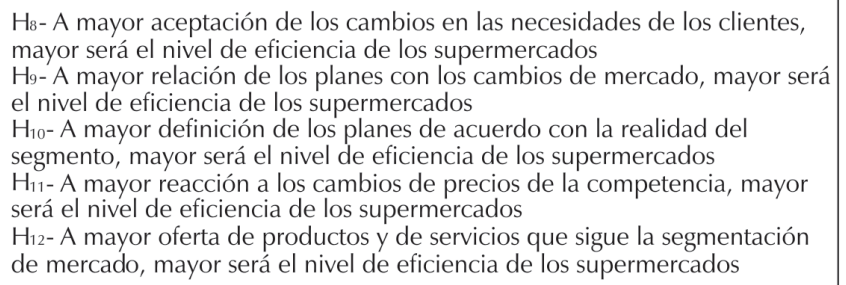 & $\begin{array}{l}\text { Rechazada } \\
\text { Rechazada } \\
\text { Rechazada } \\
\text { Rechazada } \\
\text { Rechazada }\end{array}$ \\
\hline $\begin{array}{l}\text { Implementación de } \\
\text { respuesta }\end{array}$ & $\begin{array}{l}\text { H}_{3} \text { - A mayor reacción de los supermercados a la competencia, mayor será } \\
\text { el nivel de eficiencia } \\
H_{4} \text { - A mayores acciones en contra a la insatisfacción de los clientes, mayor } \\
\text { será el nivel de eficiencia de los supermercados } \\
H_{15}-\text { A mayor oferta de nuevos servicios, mayor será el nivel de eficiencia } \\
\text { de los supermercados } \\
H_{6}-\text { A mayores acciones para establecer relaciones con los clientes, mayor } \\
\text { será el nivel de eficiencia de los supermercados }\end{array}$ & $\begin{array}{l}\text { Rechazada } \\
\text { Rechazada } \\
\text { Rechazada } \\
\text { Confirmada }\end{array}$ \\
\hline
\end{tabular}

Fuente: datos de la investigación

En términos generales, es posible verificar que los cuatro elementos de la orientación al mercado mostraron influir en la eficiencia de los supermercados, auque sea por medio de acciones específicas, lo que refleja la importancia de la práctica para el desempeño de las organizaciones y es defendido por investigadores como Day (2001), Jaworski y Kohli (1993), Narver y Slater (1990) y Panigyrakis y Theodoridis (2007).

Más allá de la contribución para el desempeño, se refuerza el hecho de que la orientación al mercado es fuente de ventaja competitiva. Hunt y Lambe (2000) consideran que la orientación al mercado consiste de una combinación sinérgica de recursos básicos y puede ser considerada una habilidad de la organización. Bajo la visión de la teoría de la ventaja de recursos, son las habilidades internas que determinan la ventaja competitiva sostenible 
(BARNEY, 1991). Luego, si una empresa es orientada al mercado y sus concurrentes no lo son, habrá una ventaja de parte de dicha empresa.

\section{Conclusiones y consideraciones finales}

Teniendo en cuenta el objetivo de analizar la relación entre orientación al mercado y eficiencia en los supermercados, es posible destacar algunas consideraciones importantes del estudio.

Inicialmente, los hallazgos permitieron verificar que la orientación al mercado ejerce un efecto positivo en la eficiencia de las empresas analizadas, por medio de acciones relacionadas a la generación y diseminación de inteligencia y a la implementación de respuesta.

Los resultados son soportados por investigaciones anteriores como la de Panigyrakis y Theodoridis (2007), que evidenciaron el efecto positivo de la orientación al mercado en el desempeño en los supermercados de Grecia. También muestran resultados positivos los estudios de Becker y Homburg (1999), Baker y Sinkula (1999a), Bhuian (1998), Kumar, Subramanian y Yauger (1998), Egeren y O'connor (1998), Jaworski y Kohli (1993) y Narver y Slater (1990). En Brasil, las investigaciones de Souza (2004), Perin, Sampaio y Faleiro (2004), Perin y Sampaio (2003) y Sampaio (2000) también mostraron el efecto positivo de la orientación al mercado en el desempeño, aunque ninguna de esas investigaciones fue desarrollada en el segmento de los supermercados o haya considerado la eficiencia como medida de desempeño.

Aunque gran parte de las investigaciones tenga comprobado la influencia benéfica de la orientación al mercado en el desempeño, parece evidente que esa relación se muestra distinta o inexistente dependiendo del contexto y del tipo de empresa bajo análisis. Kirca, Jayachandran y Bearden (2005) confirmaron ese hecho al hallar evidencias de que la relación entre la orientación al mercado y el desempeño es más intensa en las empresas productoras y más fuerte para las medidas de desempeño relativas a costes y a la renta en esas mismas empresas.

La relación observada en esa investigación indica esa tendencia pues la intensidad de la relación fue afectada por la influencia negativa de la variable referente a la concepción de respuesta. Tal vez las idiosincrasias de las organizaciones deban ser consideradas cuando se estudia el tema y sea válido desarrollar estudios de caso. Trabajos recientes están siguiendo esa orientación como es el caso de las investigaciones de Gebhardt, Carpenter y Sherry Jr. (2006) y de Rogers, Ghauri y George (2005).

Las contribuciones teóricas de esa investigación se relacionan con: la posibilidad de utilizar medidas opcionales de desempeño en la relación con orientación al mercado; y con las ventajas obtenidas por medio de la individualización de los elementos de la orientación al mercado. La eficiencia, una medida del desempeño operacional del negocio, se mostró adecuada cuando relacionada a las actitudes de orientación al mercado, lo que significa que nuevas posibilidades surgen de ese resultado. De la misma manera, la opción por la regresión de cada uno de los elementos de la orientación al mercado con la eficiencia mostró lo que de hecho influye en el desempeño.

Comprobadamente, la orientación al mercado es importante para determinar el desempeño del negocio, aunque ese desempeño haya sido considerado generalmente por medidas tradicionales como el volumen de ventas, el crecimiento de las ventas y la participación del mercado. Hasta entonces, una medida directa y resultante de la relación entre inputs y outputs no había sido considerada para el análisis. Los beneficios de esa medida significan la posibilidad de medir el impacto directo de las acciones de la orientación al mercado en las operaciones del negocio siendo esas un termómetro del amplio desempeño de las organizaciones

Rev. Adm. UFSM, Santa Maria, v. I, N. 1, P. 134-152, JAN./ABR. 2008 
(VENKATRAMAN y RAMANUJAM, 1986). También, el beneficio está en la identificación de procesos específicos y directamente relacionados a la orientación al mercado para establecer la relación. En tal sentido nuevas descubiertas pueden surgir en la verificación de esa relación en otros contextos o sectores específicos de la actividad económica.

La propuesta de traer la eficiencia para el contexto de los estudios sobre orientación al mercado, en el ámbito de la teoría de marketing, muestra también la posibilidad de aproximar dos campos teóricos poco explorados en conjunto. Aparentemente, tanto la teoría de marketing como la teoría neoclásica de la firma presentan evoluciones teóricas propias y establecer relaciones entre las dos teorías puede ser algo importante para el avance de los estudios organizacionales.

Al considerar contextos específicos para el análisis también se mostró importante la regresión individual de los elementos de la orientación al mercado con la eficiencia. La opción por ese tipo de procedimiento permitió identificar las acciones específicas de cada uno de los elementos (generación de inteligencia, diseminación de inteligencia, concepción de respuesta e implementación de respuesta) que pueden influir en la eficiencia. Los hallazgos permitieron comprender o justificar las acciones de orientación al mercado en contextos específicos de análisis, evidenciando los comportamientos característicos de las empresas y mostrando cómo esas actitudes reflejan en la orientación al mercado. Específicamente en ambientes muy competitivos, como es el caso del segmento minorista de alimentos, acciones motivadas por la rivalidad entre los concurrentes fueron evidenciadas en el ámbito de la orientación al mercado.

Para concluir, en las relaciones establecidas en el presente trabajo, fue posible descubrir que la orientación al mercado no se relaciona ampliamente con la eficiencia pues solamente algunas acciones de la orientación la influenciaron. Aunque no sea posible inferir que ese sería el mismo comportamiento si otras medidas de desempeño fueran consideradas, queda en abierto la necesidad de probar nuevamente el concepto teniendo en cuenta cada uno de sus elementos.

\section{Referencias}

ANTONI, V.L.; DAMACENA, C.; LEZANA, A.G.R. Um modelo preditivo de orientação para o mercado: um estudo no contexto do ensino superior brasileiro. In: ENCONTRO ANUAL DA ASSOCIAÇÃO NACIONAL DE PÓS-GRADUAÇÃO EM ADMINISTRAÇÃO, 28., 2004, Curitiba, PR. Anais... Curitiba: ANPAD, 2004.

APPIAH-ADU, K. market orientation and performance: empirical tests in a transition economy. Journal of Strategic Marketing, v.6, p.25-45, 1998.

APPIAH-ADU, K.; RANCHHOD, A.

Market orientation and performance in the biotechnology industry: an exploratory empirical analysis. Technology Analysis \& Strategic

Management, v.10, n.2, p.197-210, 1998.

AVLONITIS, G.J.; GOUNARIS, S.P. Marketing orientation and its determinants: an empirical analysis. European Journal of Marketing, v.33, n.11/12, p.1003-1037, 1999.

$\mathrm{BABBIE}, \mathrm{E}$. Métodos de pesquisas de survey. Belo Horizonte: Editora UFMG, 1999.

BAKER, W.E.; SINKULA, J.M. The synergistic effect of market orientation and learning orientation on organizational performance.

Journal of the Academy of Marketing Science, v.27, n.4, p.411-427, 1999a. 
BAKER, W.E.; SINKULA, J.M. Learning orientation, market orientation, and innovation: integrating and extending models of organizational performance. Journal of MarketFocused Management, v.4, p.295-308, 1999b.

BARNEY, J.B. Firm resources and sustained competitive advantage. Journal of Management, v.17, n.1, p. 99-120, 1991.

BECKER, J.; HOMBURG, C. Market-oriented management: a systems-based perspective. Journal of Market-Focused Management, v.4, p.17-41, 1999.

BEST, R.; HAWKINS, D.I.; ALBAUM, G. The role of random role of random weights and reliability in the assessment of multiattribute attitude models. Advances in Consumer Research, v. 3, n.1, p. 88-91, 1976.

BHUIAN, S.N. An empirical examination of market orientation in Saudi Arabian manufacturing companies. Journal of Business Research, v.43, p.13-25, 1998.

CASTILHO FILHO, J.P. de; AKEL SOBRINHO, Z. Orientação para o mercado em shopping center's: um estudo comparativo em shopping center's de valor e convencionais na cidade de Curitiba. In: ENCONTRO DE MARKETING, 1, 2004, Porto Alegre, RS. Anais... Porto Alegre: ANPAD, 2004.

CHARNES A.; COOPER W.W.; RHODES E. Measuring the efficiency on decision making units. European Journal of Operational Research, v. 2, p. 429-444, 1978.

$\mathrm{CHOU}, \mathrm{P}$. Modeling daily price limits. International Review of Finance Analysis, v.8, n.3, p.283-301, 1999.

DARDEN, W.R.; PERREAULT Jr, W.D. Identifying interurban shoppers: multiproduct purchase patterns and segmentation profiles. Journal of Marketing Research, v.13, n.1, p. 51-60, 1976.

DAY, G.S. A empresa orientada para o mercado. Porto Alegre: Bookman, 2001.

DAY, G.S. The capabilities of market-driven organizations. Journal of Marketing, v.58, oct., p.37-52, 1994.
DAY, G.S.; WENSLEY, R. Assessing advantage: a framework for diagnosing competitive superiority. Journal of Marketing, v.52, apr., p.1-20, 1988.

DEBREU, G. The coefficient of resource utilization. Econometrica, v.19, n.3, p. 273-292, 1951.

DENG, S.; DART, J. Measuring market orientation: a multi-factor, multi-item approach. Journal of Marketing Management, v.10, n.8, p.725-742, 1994.

DESPHANDÉ, R.; WEBSTER Jr., F.E.

Organizational culture and marketing: defining the research agenda. Journal of Marketing, v.53, jan., p.3-15, 1989.

DESPHANDÉ, R.; FARLEY, J.U.; WEBSTER Jr., F.E. Corporate culture, customer orientation, and innovativeness in Japanese firms: a quadrad analysis. Journal of Marketing, v.57, jan., p.2327, 1993.

DONTHU, N.; YOO, B. Retail productivity assessment using data envelopment analysis. Journal of Retailing, v.74, n.1, p.89-105, 1998. DUBELAAR, C.; BHARGAVA, M.; FERRARIN, D. Measuring retail productivity, what really matters? Journal of Business Research, v.55, p.417-426, 2002.

EGEREN, M.V.; O'CONNOR, S. Drivers of market orientation and performance in service firms. Journal of Services Marketing, v.12, n.1, p.39-58, 1998.

FÄRE, R.; GROSSKOPF, S.; LOVELL, C. A. K. The structure of technical efficiency. Scandinavian Journal of Economics, v.85, p.181-190, 1983.

FÄRE, R.; GROSSKOPF, S.; LOVELL, C. A. K. Production frontiers. Cambridge: Cambridge University Press, 1994.

FARRELL, M. J. The measurement of productive efficiency. Journal of the Royal Statistical Society. Series A (General), v.120, n.3, p.253290, 1957.

FARRELL, M.A.; OCZKOWSKI, E. Are market orientation and learning orientation necessary for superior organizational performance? Journal of Market-Focused Management, v.5, p.197-217, 2002. 
GEBHARDT, G.F.; CARPENTER, G.S.; SHERRY Jr., J.F. Creating a market orientation: a longitudinal, multiform, grounded analysis of cultural transformation. Journal of Marketing, v.70, oct., p.37-55, 2006.

GREENLEY, G.E. Market orientation and company performance: empirical evidence from UK companies. British Journal of Management, v.6, p.1-13, 1995.

HAIR, J.F., ANDERSON, R.E., \& TATHAM, R.L. Multivariate data analysis. 5 ed. New Jersey: Prentice Hall, 1998.

HAN, J.K.; KIM, N.; SRIVASTAVA, R.K. Market orientation and organizational performance: is innovation a missing link? Journal of Marketing, v.62, oct., p.30-45, 1998.

HOFF, A. Second stage DEA: comparison of approaches for modeling the DEA score.

European Journal of Operational Research, v. 181, n.1, aug., p.425-435, 2007.

HOOLEY, G.J.; LYNCH, J.E.; SHEPHERD, J. The market concept: putting the theory into practice. European Journal of Marketing, v.24, n.9, p.724, 1990.

HUNT, S.D.; LAMBE, J.C. Marketing's contribution to business strategy: market orientation, relationship marketing, and resource-advantage theory. International Journal of Management Reviews, v.2, n.1, p.17-44, 2000.

HUNT, S.D.; MORGAN, R.M. The comparative advantage theory of competition. Journal of Marketing, v.59, n.2, p.1-15, 1995.

INGENE, C. A. Labor productivity in retailing. Journal of Marketing, v.46, n.4, p.75-90, 1982.

JAWORSKI, B.J.; KOHLI, A.K. Market orientation: antecedents and consequences. Journal of Marketing, v.57, jul., p.53-70, 1993.

KEH, H. T; PARK, S. Y. To market, to market: the changing face of grocery retailing. Long Range Planning, v.30, n.6, p.836-846, 1997.

KERIN, R.A. In pursuit of an ideal: the editorial and literary history of the journal of marketing. Journal of Marketing, v.60, n.1, jan., p.1-13, 1996.
KIRCA, A.H.; JAYACHANDRAN, S.; BEARDEN, W.O. Market orientation: a meta-analytic review and assessment of its antecedents and impact on performance. Journal of Marketing, v.69, apr., p.24-41, 2005.

KOHLI, A.K.; JAWORSKI, B.J. Market orientation: the construct, research propositions, and managerial implications. Journal of Marketing, v.54, April, p.1-18, 1990.

KOOPMANS, T.C. Efficient allocation of resources. Econometrica, v.19, n.4, p.455-465, 1951.

KUMAR, K.; SUBRAMANIAN, R.; YAUGER, C. Examining the market orientation-performance relationship. Journal of Management, v.24, n.2, p.201-233, 1998.

LIN, B.W.; CHEN, J. S. Corporate technology portfolios and R\&D performance measures: a study of technology intensive firms. R\&D Management, v.35, n.2, p.157-170, 2005.

LIU, H.; DAVIES, G. Market orientation in UK multiple retail companies: nature and pattern. International Journal of Service Industry Management, v.8, n.2, p.170-187, 1997.

LYNCH, D.F; KELLER, S.B; OZMENT, J. The effects of logistics capabilities and strategy on firm performance. Journal of Business Logistics, v.21, n.2, p.47-67, 2000.

MACERA, A.P.; URDAN, A.T. (2002), Orientação para o Mercado externo: teste de um modelo no Brasil e sua aplicação a uma amostra de empresas exportadoras brasileiras. In: ENCONTRO DA ASSOCIAÇÃO NACIONAL DE PÓS-GRADUAÇÃO EM ADMINISTRAÇÃO, 26, 2002, Salvador, BA. Anais... Salvador: ANPAD, 2002.

MALHOTRA, N. Pesquisa de marketing: uma orientação aplicada. 3 ed. Porto Alegre: Bookman, 2001.

MATTAR, F.N. Pesquisa de marketing. v.1. 5 ed. São Paulo: Atlas, 1999.

McNAMARA, C.P. The present status of the marketing concept. Journal of Marketing, v.36, jan., p.50-57, 1972.

MELLO, S.C.B. de; SOUZA, A.C.R. de. 
Purificação e validação da escala de orientação para o mercado de George DAY. In: ENCONTRO DA ASSOCIAÇÃO NACIONAL DE PÓSGRADUAÇÃO EM ADMINISTRAÇÃO, 27, 2003, Atibaia, SP. Anais... Atibaia: ANPAD, 2003.

NARVER, J.C.; SLATER, S.F. The effect of a market orientation on business profitability. Journal of Marketing, oct., p.20-35, 1990.

NARVER, J.C.; SLATER, S.F.; TIETJE, B. Creating a marketing orientation. Journal of Market Focused Management, v.2, p.241-255, 1998.

NUNNALLY, J.C. Introducción a la medición psicológica. Buenos Aires: Paidos, 1970.

PANIGYRAKIS, G.G.; THEODORIDIS, P.K. Market orientation and performance: an empirical investigation in the retail industry in Greece. Journal of Retailing and Consumer Services, v.14, p.137-149, 2007.

PÉREZ, C. Técnicas estadísticas con SPSS. Madrid: Pearson Educación S.A, 2001.

PERIN, M.G.; SAMPAIO, C.H. Orientação para o mercado, porte empresarial e performance: um estudo longitudinal no setor eletro-eletrônico do Brasil. In: ENCONTRO DA ASSOCIAÇÃO NACIONAL DE PÓS-GRADUAÇÃO EM ADMINISTRAÇÃO, 27, 2003, Atibaia, SP. Anais... Atibaia: ANPAD, 2003.

PERIN, M.G.; SAMPAIO, C.H.; FALEIRO, S.N. O impacto da orientação para o mercado e da orientação para a aprendizagem sobre a inovação de produto: uma comparação entre a indústria eletroeletrônica e o setor de ensino universitário de administração. Revista de Administração Contemporânea, v.8, n.1, jan./ mar., p.79-103, 2004.

PHILLIPS, P.A.; DAVIES, F.; MOUTINHO, L. Assessing the impact of market-focused and price-based strategies on performance: a neural network typology. Journal of Market-Focused Management, v.5, p.219-238, 2002.

PITT, L.; CARUANA, A.; BERTHON, P.R. Market orientation and business performance: some European evidence. International Marketing Review, v.13, n.1, p.5-18, 1996.

ROGERS, H.; GHAURI, P.N.; GEORGE, K.L.
The impact of market orientation on the internationalization of retailing firms: Tesco in eastern Europe. International Review of Retail, Distribution and Consumer Research, v.15, n.1, jan., p.53-74, 2005.

SALAS, V. F. Economía teórica de la empresa. Información Comercial Española, n.611, Julio, p.9-22, 1984.

SAMPAIO, C.H. Relação entre orientação para o mercado e performance empresarial em empresas de varejo de vestuário do Brasil. 2000. Tese (Doutorado em Administração) Escola de Administração, Universidade Federal do Rio Grande do Sul, Porto Alegre, 2000.

SARGEANT, A.; MOHAMAD, M. Business performance in the UK hotel sector - does it pay to be market oriented? The Service Industries Journal, v.19, n.3, jul., p.42-59, 1999.

SCHENDEL, D.E.; PATHON, G.R. A simultaneous equation model of corporate strategy.

Management Science, v.24, n.15, Nov., p.16111621, 1978.

SHAPIRO, B.P. What the Hell is market oriented? Harvard Business Review, nov.-dec., p.119125, 1988.

SLATER, S.F.; NARVER, J.C. Does competitive environment moderate the market orientationperformance relationship? Journal of Marketing, jan., p.46-55, 1994.

SLATER, S.F.; NARVER, J.C. Market orientation and the learning organization. Journal of Marketing, v.59, jul., p.63-74, 1995.

SOUZA, A.C.R. de. A orientação para o mercado, o relacionamento com os clientes e o desempenho das empresas: um levantamento junto a empresas que atuam na região nordeste do Brasil. In: ENCONTRO DA ASSOCIAÇÃO NACIONAL DE PÓS-GRADUAÇÃO EM ADMINISTRAÇÃO, 28, 2004, Curitiba, PR. Anais... Curitiba: ANPAD, 2004.

TOBIN, J. Estimation of relationship for limited dependent variables. Econometrica, v.26, n.1, p.24-36, 1958.

VENKATRAMAN, N.; RAMANUJAM, V. 
ORIENTACIÓN AL MERCADO...

Measurement of business performance in strategy research: a comparison of approaches. Academy of Management Review, v.1, n.4, p.801-814, 1986.

WALKER, O.C.; RUEKERT, R.W. Marketing's role in the implementation of business strategies: a critical review and conceptual framework.

Journal of Marketing, v.51, jul., p.15-33, 1987.

ZHOU, K.Z.; YIM, C.K.B.; TSE, D.K. The effects of strategic orientations on technology and market-based breakthrough innovations. Journal of Marketing, v.69, apr., p.42-60, 2005. 